CUBO A Mathematical Journal Vol.12, № 03, (13-32). October 2010

\title{
A Family of Stationary Solutions to the Euler Equations and Generalized Solutions
}

\author{
Juliana Conceição Precioso \\ Departamento de Matemática, \\ Universidade Estadual Paulista, \\ 15054-000, S.J.R. Preto, SP, Brazil \\ email: precioso@ibilce.unesp.br
}

\begin{abstract}
In this work, we present a interesting family of stationary solutions for the Euler equations, which behaves in the same way that the approximated solutions presented in [6].

\section{RESUMEN}

En este trabajo, presentamos una familia interesante de soluciones estacionarias para las ecuaciones de Euler, que se comportan de la misma manera que las soluciones aproximadas presentadas en [6].
\end{abstract}

Key words and phrases: Euler equations, incompressible flows, generalized solutions.

Math. Subj. Class.: 35D99. 


\section{Introduction}

An ideal incompressible fluid moving inside $D \subset \mathbb{R}^{n}$ is classically described by a velocity field $u(t, x)$ and a pressure field $p(t, x)$, subject to the classical Euler equations:

$$
\left\{\begin{array}{l}
\partial_{t} u+(u \cdot \nabla) u+\nabla p=0 \\
\nabla \cdot u=0
\end{array}\right.
$$

with the boundary condition that $u$ is tangent to $\partial D$.

In classical continuous mechanics [1], the motion of an incompressible inviscid fluid in a compact domain $D \subset \mathbb{R}^{n}$ can be seen as a geodesic on the group of all diffeomorphisms of $D$ with unit jacobian determinant, $G(D)$. This set is included in $S(D)$ the semigroup of all Borel maps $h$ of $D$ that satisfy

$$
\int_{D} f(h(x)) d x=\int_{D} f(x) d x, \quad \forall f \in C^{0}(D) .
$$

For more details see [1], [2] or [6].

We will denote

$$
\begin{gathered}
V:=\left\{u:[0, T] \times D \longrightarrow \mathbb{R}^{n} \text { such that } u \in C^{0}(Q), u(t, \cdot) \in \operatorname{Lip}(D)\right. \\
\text { uniformly in } \left.0 \leq t \leq T, \operatorname{div} u=0, u(t, \cdot) \cdot \widehat{n}_{\left.\right|_{\partial D}}=0\right\}
\end{gathered}
$$

Note that the flow $(t, x) \mapsto g(t, x)$ describing the motion of fluid particles is defined by

$$
\left\{\begin{array}{l}
\partial_{t} g(t, x)=u(t, g(t, x)) \\
g(0, x)=x
\end{array}\right.
$$

By Cauchy-Lipschitz theorem, for each $u \in V$, there is a unique solution to (1.2) and for each time $t$ the map $g(t, x)=g(t, \cdot) \in G(D)$. Then, by elementary calculations, the Euler equations can be replaced by the following equivalent equations:

$$
\left\{\begin{array}{l}
\partial_{t t}^{2} g(t, x)+\nabla p(t, g(t, x))=0 \\
\operatorname{det} D_{x} g(t, x)=1
\end{array}\right.
$$

Lets call (1.3) by the "Lagrangian formulation" of the Euler equations.

From a geometrical point of view, different from the natural PDE point of view which consists in adressing the Euler equations as an evolution equation with prescribed initial 
velocity field, it is natural to solve the problem to minimize the action

$$
A(g)=\frac{1}{2} \int_{0}^{T} \int_{D}\left|\partial_{t} g(t, x)\right|^{2} d x d t
$$

among all trajectories on $G(D)$ connecting $g(0, \cdot)=I d$ and $g(T, \cdot)=h$.

The corresponding system of PDE's is the lagrangian formulation of the Euler equations (1.3).

Ebin and Marsden showed local existence and uniqueness for this problem, namely, if $h$ and $I$ are sufficiently close in a sufficiently high order Sobolev norm, then there is a unique geodesic connecting $I d$ to $h$, see [9]. In the large, uniqueness can fail. However, in [12], A. I. Shnirelman shows that existence of minimal geodesics may fail to a class of data.

To solve the problem to find minimal geodesics in a generalized sense, in particular for data $h$ in Shnirelman class, was introduced suitable "Young measure", (see [15] and [18]) of different ways as in [4], [6], [12] and [13]. In [4], was used a concept that takes into account the dynamics of the particles. To each path $t \in[0, T] \longmapsto z(t) \in D$, one associates the probability that it is followed by some material particle. More precisely, was proposed a notion of the generalized flow, as been a probability measure on set $\Omega=D^{[0, T]}$ of all curves $t \in[0, T] \longrightarrow z(t) \in D$, namely, a Borel probability measure $\mu$, on product space $\Omega=D^{[0, T]}$, such that each projection $\mu_{t}$ for $0 \leq t \leq T$ is a Lebesgue measure on $D$. The action in this context is express by

$$
\int_{\Omega} \int_{0}^{T} \frac{1}{2}\left|z^{\prime}(t)\right|^{2} d \mu_{t}(z) d t
$$

Brenier showed the existence of generalized solutions and, later in [5], the existence and uniqueness of the pressure gradient linked to them through a suitable Poisson equation, but did not obtain for them a complete set of equations beyond the classical Euler equations. However, in [6] it was possible. The problem to find minimal geodesics was reformulated in terms of a pair of measures associated to the field $u$, solution of the Euler equations, in the following way: Given a smooth trajectory $t \in[0, T] \mapsto g(t, x) \in G(D)$, we define the measures (respectively nonnegative and vector-valued)

$$
c(t, x, a)=\delta(x-g(t, a)), \quad m(t, x, a)=\partial_{t} g(t, a) \delta(x-g(t, a)),
$$

defined on $Q^{\prime}=[0, T] \times D \times D$. These measures satisfy

$$
\begin{gathered}
\int_{D} c(t, x, a) d a=1, \\
\partial_{t} c+\nabla_{x} \cdot m=0, \\
c(0, x, a)=\delta(x-a) ; \quad c(T, x, a)=\delta(x-h(a)) .
\end{gathered}
$$


Moreover, the measure $m$ is absolutely continuous with respect to $c$, with a density $v \in$ $L^{2}\left(Q^{\prime}, d c\right)$, so that $m=c v$, and the action is given by

$$
A(g)=\frac{1}{2} \int_{0}^{1} \int_{D}|v(t, x, a)|^{2} c(t, x, a) d x d a
$$

or equivalently, $A(g)=K[c, m]$, where

$$
K[c, m]:=\sup _{X}\{\langle c, F\rangle+\langle m, \Phi\rangle\},
$$

where $(c, m)$ is of the form (1.4) and

$$
X=\left\{(F, \Phi) \in C^{0}\left(Q^{\prime}\right) \times\left(C^{0}\left(Q^{\prime}\right)\right)^{n} ; F(t, x, a)+\frac{1}{2}|\Phi(t, x, a)|^{2} \leq 0\right\} .
$$

Then, Brenier defined the relaxed problem, as the problem to look for pairs of measures $(c, m)$ that minimize $K[c, m]$ and are admissible in the sense of (1.5), (1.6) and (1.7), but do not necessarily satisfy (1.4).

Also was showed that, for $D=[0,1]^{n}$ and each data $h \in S(D)$, the relaxed problem always has solutions $(c, m)$ and that there is a unique locally bounded measure $\nabla_{x} p$ in the interior of $Q=[0, T] \times D$, depending only $h$, such that

$$
\partial_{t}(c v)+\nabla_{x}(c v \otimes v)+\underline{c} \nabla_{x} p=0
$$

holds in the sense of distributions on the interior of $Q^{\prime}$, where $\underline{c}$ is a extension of $c$ for which the product $\underline{c}(t, x, a) \nabla_{x} p(t, x)$ is well-defined. Moreover, was showed that for any $h \in S\left([0,1]^{3}\right)$ of the form

$$
h\left(x_{1}, x_{2}, x_{3}\right)=\left(H\left(x_{1}, x_{2}\right), x_{3}\right)
$$

that, for any $\varepsilon>0$, there is a $u_{\varepsilon} \in V$ such that

$$
K\left(u_{\varepsilon}\right)+\frac{1}{2 \varepsilon}\left\|g_{u_{\varepsilon}}(T, \cdot)-h\right\|_{L^{2}(D)}^{2} \leq I_{\varepsilon}(h)+\varepsilon,
$$

where $I_{\varepsilon}(h)=\inf _{u \in v}\left\{K(u)+\frac{1}{2 \varepsilon}\left\|g_{u}(T, \cdot)-h\right\|_{L^{2}(D)}^{2}\right\}$ and

$$
k\left(u_{\varepsilon}\right)=\frac{1}{2} \int_{0}^{T} \int_{D}\left|u_{\varepsilon}(t, x)\right|^{2} d x d t=\frac{1}{2} \int_{0}^{T} \int_{D}\left|\partial_{t} g_{\varepsilon}(t, x)\right|^{2} d x d t=A\left(g_{\varepsilon}\right) .
$$

In addition, the measures $\left(c_{\varepsilon}, m_{\varepsilon}\right)$ associated with $u_{\varepsilon}$, through (1.4), converge, as $\varepsilon \rightarrow 0$ to the generalized solutions of the relaxed problem. Moreover, the fields $u_{\varepsilon}$ satisfy

$$
\nabla_{x} \cdot u_{\varepsilon}=0, \quad \partial_{t} u_{\varepsilon}+\left(u_{\varepsilon} \cdot \nabla\right) u_{\varepsilon} \rightarrow-\nabla p
$$


weakly, as $\varepsilon$ tends to zero. As observed in [6], with each solution $(c, m)$ we may associated a measure-valued solution $\mu$, in the sense of DiPerna and Majda, by setting

$$
\int_{Q \times \mathbb{R}^{d}} f(t, x, \xi) d \mu(t, x, \xi)=\int_{Q^{\prime}} f(t, x, v(t, x, a)) d c(t, x, a),
$$

for any continuous function $f \in Q \times \mathbb{R}^{d}$ with at most quadratic growth as $\xi \rightarrow \infty$. For more details, see [6] and [7].

In [4], Brenier shows explicit examples of non trivial generalized solutions. A typical example is when $D$ is the unit disk in $2 D$ and $h(x)=-x$. We know that the problem of the minimal action has two trivial solutions $g_{+}(t, x)=e^{i \pi t} x$ and $g_{-}(t, x)=e^{-i \pi t} x$ with the same pressure field $p(x)=\frac{\pi^{2}|x|^{2}}{2}$. We have another (generalized) solution $(c, m)$ to the same problem which is given by

$$
\begin{gathered}
\int_{Q^{\prime}} f(t, x, a) c(t, x, a) d t d x d a=\int_{[0,1] \times D} \int_{0}^{1} f(t, G(t, a, \theta), a) d \theta d t d a, \\
\int_{Q^{\prime}} f(t, x, a) m(t, x, a) d t d x d a=\int_{[0,1] \times D} \int_{0}^{1} \partial_{t} G(t, a, \theta) f(t, G(t, a, \theta), a) d \theta d t d a,
\end{gathered}
$$

for all continuous function $f$, where

$$
G(t, \theta, a)=a \cos (\pi t)+\left(1-|a|^{2}\right)^{\frac{1}{2}} e^{2 i \pi \theta} \sin (\pi t) \in D .
$$

Note that each particle initially located at $a \in D$ splits up along a circle of radius $\left(1-|a|^{2}\right)^{\frac{1}{2}}$ $\sin (\pi t)$, with center $a \cos (\pi t)$, that moves across the unit disk and shrinks down to the point $-a$ when $t=1$.

In general, is very difficult to obtain explicit examples of non trivial generalized solutions and the explicit examples constructed by Brenier, are based on the model presented in [4], which takes in account a concept purely Lagrangian of Young measures, the so-called generalized flows. Beyond supplying an application of the model developed in [6], the results of this paper give an interesting information for the limit of a sequence of the stationary solutions, showing that they are associated with measures that satisfy the Euler equations in a specified weak sense. For another point of view, the results of the paper give example of as a sequence of highly oscillatory solutions still can have a limit that is solution in some sense. Namely, we exhibited a family $u_{\varepsilon}$ (which behavior as the"approximated solutions" argued above) such that when $\varepsilon \rightarrow 0$, the velocity field gets more and more oscillatory, but the measures $\left(c_{\varepsilon}, m_{\varepsilon}\right)$ associated to the field $u_{\varepsilon}$ converges to a solution $(c, m)$ of the equations

$$
\int_{D} c(t, x, a) d a=1, \quad \partial_{t} c+\nabla_{x} \cdot m=0
$$


and

$$
\partial_{t}(c v)+\nabla_{x}(c v \otimes v)+c \nabla_{x} p=0 .
$$

Equivalent vector fields to those ones treaties in this work have been studied as application of high order essentially no oscillatory (ENO) schemes for smooth solutions of NavierStokes and Euler equations, (see [14]), in problems involving the Taylor-Green vortex, (see [8], [3] and [10]) and to explore a discrete singular convolution algorithm (DSC) for solving certain mechanics problems, (see [16] and [17]).

\section{A Family of (Stationary) Solutions}

In this work we consider the following family of stationary solutions to the Euler equations:

$$
\begin{gathered}
u_{n}(x, y)=\left(-\cos (x) \sin (n y), \frac{1}{n} \sin (x) \cos (n y), 0\right), \\
p_{n}(x, y)=-\frac{1}{4}\left(\cos (2 x)+\frac{1}{n^{2}} \cos (2 n y)\right) .
\end{gathered}
$$

Note that, $\left|D p_{n}(x, y)\right| \leq C$, and when $n$ goes to infinity the pressure field strongly converges to $p(x, y)=-\frac{1}{4} \cos (2 x)$. Then, is easy verify that $\left(u_{n} \cdot \nabla\right) u_{n}+\nabla p \rightarrow 0$, when $n \rightarrow \infty$.

For a moment, let us observe the behavior of family $u_{n}$. For $n=1$ we have,

$$
u_{1}(x, y)=(-\cos (x) \sin (y), \sin (x) \cos (y), 0)
$$

and

$$
\left\{\begin{array}{l}
\dot{x}=-\cos (x) \sin (y) \\
\dot{y}=\sin (x) \cos (y) .
\end{array}\right.
$$

Then, we have $(\dot{x}, \dot{y})=(0,0) \Leftrightarrow(x, y)=\left((2 k+1) \frac{\pi}{2},(2 l+1) \frac{\pi}{2}\right)$ or $(k \pi, l \pi)$, where $k, l \in \mathbb{Z}$, (see Figure 1).

For $n=2$ we have, $(\dot{x}, \dot{y})=(0,0) \Leftrightarrow(x, y)=\left((2 k+1) \frac{\pi}{2},(2 l+1) \frac{\pi}{4}\right)$ or $\left(k \pi, \frac{l \pi}{2}\right)$, where $k, l \in \mathbb{Z}$, (see Figure 2).

Thus, for $n$ we have, $(\dot{x}, \dot{y})=(0,0) \Leftrightarrow(x, y)=\left((2 k+1) \frac{\pi}{2},(2 l+1) \frac{\pi}{2 n}\right)$ or $\left(k \pi, \frac{l \pi}{n}\right)$, where $k, l \in \mathbb{Z}$.

Note that, when $n \rightarrow \infty$ the velocity field gets more and more oscillatory. In the next section we will show that the measures $\left(c_{n}, m_{n}\right)$ defined by $c_{n}(t, x, a)=\delta\left(x-g_{u_{n}}(t, a)\right), m_{n}(t, x, a)=$ $u_{n}(t, x) \delta\left(x-g_{u_{n}}(t, a)\right)$ converges to the solution $(c, m)$ of the equations:

$$
\int_{D} c(t, x, a) d a=1
$$




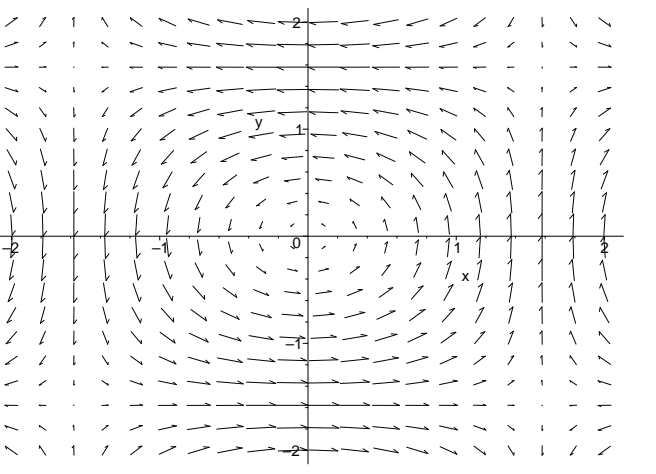

Figure 1: Phase portrait of the velocity field $u_{1}(x, y)=(-\cos (x) \sin (y), \sin (x) \cos (y), 0)$

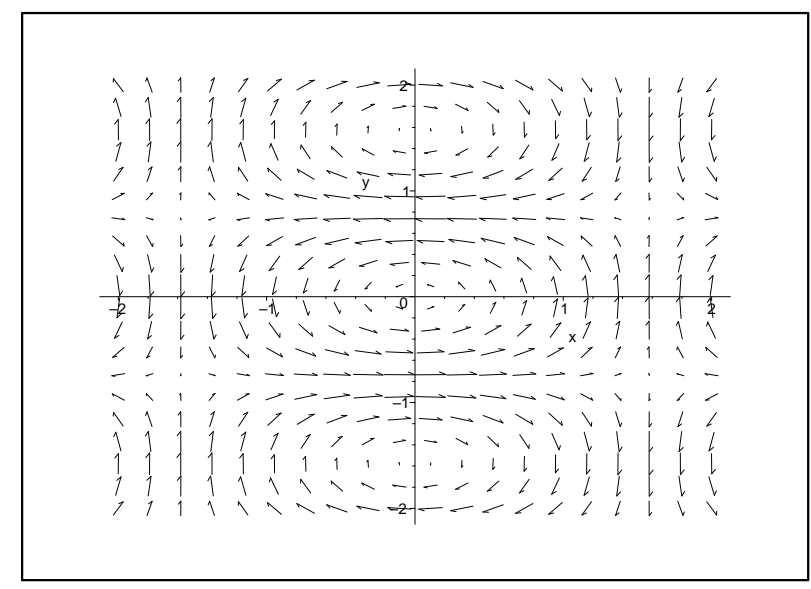

Figure 2: Phase portrait of the velocity field $u_{2}(x, y)=\left(-\cos (x) \sin (2 y), \frac{1}{2} \sin (x) \cos (2 y), 0\right)$

$$
\begin{gathered}
\partial_{t} c+\nabla_{x} \cdot m=0 \\
\partial_{t}(c v)+\nabla_{x} \cdot(c v \otimes v)+\underline{c} \nabla_{x} p=0,
\end{gathered}
$$

By the consistency theorem in [6], or its generalization for variable density in [11], we know that if $u_{n}$ is a solution of the Euler equations, then the pair of the measures $\left(c_{n}, m_{n}\right)$ defined as below satisfy the equations (2.2), (2.3), (2.4). 


\section{The Limite $(c, m)$}

In this section we build explicitly the limite $(c, m)$. For this, in first we rewrite the field

$$
u_{n}(x, y)=\left(-\cos (x) \sin (n y), \frac{1}{n} \sin (x) \cos (n y), 0\right)
$$

as

$$
u_{n}(x, y)=\left(u_{1}^{1}(x, n y), \frac{1}{n} u_{1}^{2}(x, n y), 0\right)
$$

where

$$
u_{1}^{1}(x, n y)=-\cos (x) \sin (n y) \quad \text { and } \quad u_{1}^{2}(x, n y)=\sin (x) \cos (n y) .
$$

Of here in ahead, we will omit third coordinate of the fields $u_{n}^{\prime}$ s. We also observe that the field has period $2 \pi$. Now, we define

$$
\left\{\begin{array}{l}
x=x(t, \gamma, \delta) \\
y=y(t, \gamma, \delta)
\end{array}\right.
$$

the solution of

$$
\left\{\begin{array}{l}
\frac{d x}{d t}=u_{1}^{1}(x, y)=\cos (x) \sin (y) \\
\frac{d y}{d t}=u_{1}^{2}(x, y)=\sin (x) \cos (y) \\
x(0, \gamma, \delta)=\gamma \\
y(0, \gamma, \delta)=\delta
\end{array}\right.
$$

Let be $0 \leq i \leq n-1,0 \leq \alpha_{1} \leq 2 \pi$, and $\frac{2 \pi i}{n} \leq \alpha_{2} \leq \frac{2 \pi}{n}(i+1)$, where $i, n \in \mathbb{N}$. This is:

- for $n=1, i=0$ and we have $0 \leq \alpha_{2} \leq 2 \pi$.

- for $n=2,0 \leq i \leq 1$ and we have

$$
\left\{\begin{array}{l}
0 \leq \alpha_{2} \leq \pi, \text { if } \mathrm{i}=0 \\
\pi \leq \alpha_{2} \leq 2 \pi, \text { if } \mathrm{i}=1
\end{array}\right.
$$

- for $n=k, 0 \leq i \leq k-1$ and we have

$$
\left\{\begin{array}{l}
0 \leq \alpha_{2} \leq \frac{2 \pi}{k}, \text { if } \mathrm{i}=0 \\
\frac{2 \pi}{k} \leq \alpha_{2} \leq \frac{4 \pi}{k}, \text { if } \mathrm{i}=1 \\
\ldots \\
\frac{2 \pi(k-1)}{k} \leq \alpha_{2} \leq 2 \pi, \text { if } \mathrm{i}=(\mathrm{k}-1) .
\end{array}\right.
$$



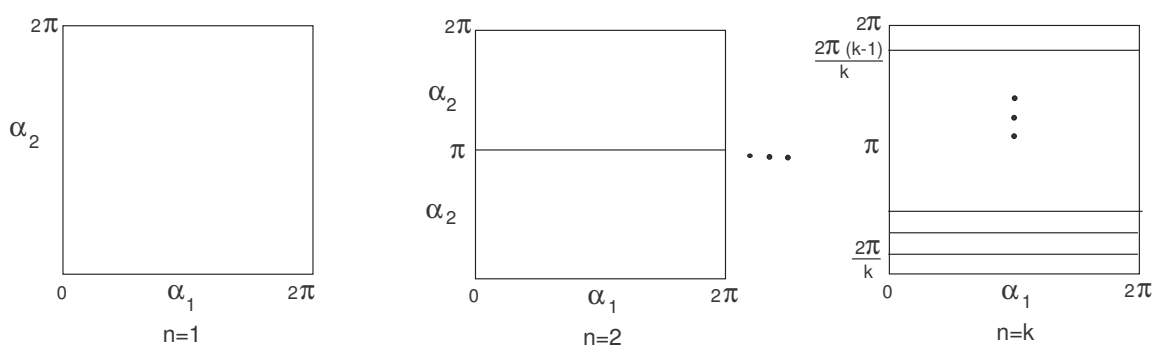

Figure 3:

Then, $i$ counts the cells (in the vertical line) from 0 to $2 \pi$ for each $n$, as we can observe in Figure 3.

Now, we define

$$
\left\{\begin{array}{l}
x_{n}^{i}\left(t, \alpha_{1}, \alpha_{2}\right):=x\left(t, \alpha_{1}, n\left(\alpha_{2}-\frac{2 \pi i}{n}\right)\right) \\
y_{n}^{i}\left(t, \alpha_{1}, \alpha_{2}\right):=\frac{1}{n} y\left(t, \alpha_{1}, n\left(\alpha_{2}-\frac{2 \pi i}{n}\right)\right)+\frac{2 \pi i}{n} .
\end{array}\right.
$$

Note that, by the definition above

- for $n=1$, we have $i=0$, thus

$$
\left\{\begin{array}{l}
x_{1}^{0}\left(t, \alpha_{1}, \alpha_{2}\right):=x\left(t, \alpha_{1}, \alpha_{2}\right), \\
y_{1}^{0}\left(t, \alpha_{1}, \alpha_{2}\right):=y\left(t, \alpha_{1}, \alpha_{2}\right) .
\end{array}\right.
$$

- for $n=2$, we have $0 \leq i \leq 1$, thus

$$
\left\{\begin{array}{c}
x_{2}^{0}\left(t, \alpha_{1}, \alpha_{2}\right):=x\left(t, \alpha_{1}, 2 \alpha_{2}\right) \\
y_{2}^{0}\left(t, \alpha_{1}, \alpha_{2}\right):=\frac{1}{2} y\left(t, \alpha_{1}, 2 \alpha_{2}\right)
\end{array} \text {,if } \mathrm{i}=0\right.
$$

and

$$
\left\{\begin{array}{l}
x_{2}^{1}\left(t, \alpha_{1}, \alpha_{2}\right):=x\left(t, \alpha_{1}, 2\left(\alpha_{2}-\pi\right)\right) \\
y_{2}^{1}\left(t, \alpha_{1}, \alpha_{2}\right):=\frac{1}{2} y\left(t, \alpha_{1}, 2\left(\alpha_{2}-\pi\right)\right)+\pi
\end{array} \text {,if } \mathrm{i}=1 .\right.
$$

- for $n=k$, we have $0 \leq i \leq k-1$, thus

$$
\left\{\begin{array}{l}
x_{k}^{0}\left(t, \alpha_{1}, \alpha_{2}\right):=x\left(t, \alpha_{1}, k \alpha_{2}\right) \\
y_{k}^{0}\left(t, \alpha_{1}, \alpha_{2}\right):=\frac{1}{k} y\left(t, \alpha_{1}, k \alpha_{2}\right)
\end{array} \text {,if } \mathrm{i}=0,\right.
$$




$$
\left\{\begin{array}{c}
x_{k}^{k-1}\left(t, \alpha_{1}, \alpha_{2}\right):=x\left(t, \alpha_{1}, k\left(\alpha_{2}-\frac{2(k-1) \pi}{k}\right)\right) \\
y_{2}^{k-1}\left(t, \alpha_{1}, \alpha_{2}\right) \\
:=\frac{1}{k} y\left(t, \alpha_{1}, k\left(\alpha_{2} \frac{2(k-1) \pi}{k}\right)\right)+\frac{2(k-1) \pi}{k}
\end{array} \text {, if } \mathrm{i}=\mathrm{k}-1 .\right.
$$

Then, we conclude that $0 \leq x_{n}^{i} \leq 2 \pi, \frac{2 \pi i}{n} \leq y_{n}^{i} \leq \frac{2 \pi(i+1)}{n}$ and

$$
\left\{\begin{array}{l}
x_{n}^{i}\left(0, \alpha_{1}, \alpha_{2}\right)=x\left(0, \alpha_{1}, n\left(\alpha_{2}-\frac{2 \pi i}{n}\right)\right)=\alpha_{1} \\
y_{n}^{i}\left(0, \alpha_{1}, \alpha_{2}\right)=\frac{1}{n} y\left(0, \alpha_{1}, n\left(\alpha_{2}-\frac{2 \pi i}{n}\right)\right)+\frac{2 \pi i}{n}=\alpha_{2} .
\end{array}\right.
$$

Moreover, by (3.2) we have

$$
\left\{\begin{array}{l}
\frac{d x_{n}^{i}}{d t}\left(t, \alpha_{1}, \alpha_{2}\right)=\frac{d x}{d t}\left(t, \alpha_{1}, n\left(\alpha_{2}-\frac{2 \pi i}{n}\right)\right) \\
\frac{d y_{n}^{i}}{d t}\left(t, \alpha_{1}, \alpha_{2}\right)=\frac{1}{n} \frac{d y}{d t}\left(t, \alpha_{1}, n\left(\alpha_{2}-\frac{2 \pi i}{n}\right)\right)
\end{array}\right.
$$

Therefore, by (3.1) and (3.3)

$$
\begin{aligned}
\frac{d x_{n}^{i}}{d t} & =u_{1}^{1}\left(x\left(t, \alpha_{1}, n\left(\alpha_{2}-\frac{2 \pi i}{n}\right)\right), y\left(t, \alpha_{1}, n\left(\alpha_{2}-\frac{2 \pi i}{n}\right)\right)\right) \\
& =u_{1}^{1}\left(x_{n}^{i}\left(t, \alpha_{1}, \alpha_{2}\right), n y_{n}^{i}\left(t, \alpha_{1}, \alpha_{2}\right)-2 \pi i\right) \\
& =u_{1}^{1}\left(x_{n}^{i}\left(t, \alpha_{1}, \alpha_{2}\right), n y_{n}^{i}\left(t, \alpha_{1}, \alpha_{2}\right)\right) \\
& =u_{n}^{1}\left(x_{n}^{i}\left(t, \alpha_{1}, \alpha_{2}\right), y_{n}^{i}\left(t, \alpha_{1}, \alpha_{2}\right)\right)
\end{aligned}
$$

and

$$
\begin{aligned}
\frac{d y_{n}^{i}}{d t} & =\frac{1}{n} u_{1}^{2}\left(x\left(t, \alpha_{1}, n\left(\alpha_{2}-\frac{2 \pi i}{n}\right)\right), y\left(t, \alpha_{1}, n\left(\alpha_{2}-\frac{2 \pi i}{n}\right)\right)\right) \\
& =\frac{1}{n} u_{1}^{2}\left(x_{n}^{i}\left(t, \alpha_{1}, \alpha_{2}\right), n y_{n}^{i}\left(t, \alpha_{1}, \alpha_{2}\right)-2 \pi i\right) \\
& =\frac{1}{n} u_{1}^{2}\left(x_{n}^{i}\left(t, \alpha_{1}, \alpha_{2}\right), n y_{n}^{i}\left(t, \alpha_{1}, \alpha_{2}\right)\right) \\
& =u_{n}^{2}\left(x_{n}^{i}\left(t, \alpha_{1}, \alpha_{2}\right), y_{n}^{i}\left(t, \alpha_{1}, \alpha_{2}\right)\right) .
\end{aligned}
$$

Now defining

$$
\left\{\begin{array}{l}
x_{n}\left(t, \alpha_{1}, \alpha_{2}\right):=x_{n}^{i}\left(t, \alpha_{1}, \alpha_{2}\right) \\
y_{n}\left(t, \alpha_{1}, \alpha_{2}\right):=y_{n}^{i}\left(t, \alpha_{1}, \alpha_{2}\right)
\end{array} \text { if } \frac{2 \pi \mathrm{i}}{\mathrm{n}} \leq \alpha_{2} \leq \frac{2 \pi(\mathrm{i}+1)}{\mathrm{n}}\right.
$$


we conclude that

$$
\left\{\begin{array}{l}
\frac{d x_{n}}{d t}\left(t, \alpha_{1}, \alpha_{2}\right)=u_{n}^{1}\left(x_{n}\left(t, \alpha_{1}, \alpha_{2}\right), y_{n}\left(t, \alpha_{1}, \alpha_{2}\right)\right) \\
\frac{d y_{n}}{d t}\left(t, \alpha_{1}, \alpha_{2}\right)=u_{n}^{2}\left(x_{n}\left(t, \alpha_{1}, \alpha_{2}\right), y_{n}\left(t, \alpha_{1}, \alpha_{2}\right)\right) \\
x_{n}\left(0, \alpha_{1}, \alpha_{2}\right)=\alpha_{1} \\
y_{n}\left(0, \alpha_{1}, \alpha_{2}\right)=\alpha_{2} .
\end{array}\right.
$$

In the remain of the work, for simplicity, we will use the following notation: $D_{i}=(0,2 \pi)^{i}=$ $(0,2 \pi) \times \cdots \times(0,2 \pi), i$ times, where $i=1, \cdots, 4$. Now, we are ready to show the following result:

Theorem 3.1. Consider $\left(x_{n}, y_{n}\right)$ solution of (3.4). Let

$$
\left\{\begin{array}{l}
c_{n}\left(t, x, y, \alpha_{1}, \alpha_{2}\right)=\delta\left((x, y)-\left(x_{n}\left(t, \alpha_{1}, \alpha_{2}\right), y_{n}\left(t, \alpha_{1}, \alpha_{2}\right)\right)\right) \\
m_{n}\left(t, x, y, \alpha_{1}, \alpha_{2}\right)=u_{n}(x, y) \delta\left((x, y)-\left(x_{n}\left(t, \alpha_{1}, \alpha_{2}\right), y_{n}\left(t, \alpha_{1}, \alpha_{2}\right)\right)\right) .
\end{array}\right.
$$

Then,

$$
\left\langle\varphi, c_{n}\right\rangle \rightarrow \frac{1}{2 \pi} \int_{D_{3}} \int_{0}^{T} \varphi\left(x\left(t, \alpha_{1}, \beta_{2}\right), \gamma, \alpha_{1}, \gamma, t\right) d t d \alpha_{1} d \beta_{2} d \gamma
$$

and $\left\langle\phi, m_{n}\right\rangle \rightarrow$

$$
\frac{1}{2 \pi} \int_{D_{3}} \int_{0}^{T} \phi^{1}\left(x\left(t, \alpha_{1}, \beta_{2}\right), \gamma, \alpha_{1}, \gamma, t\right) u_{1}^{1}\left(x\left(t, \alpha_{1}, \beta_{2}\right), y\left(t, \alpha_{1}, \beta_{2}\right)\right) d t d \alpha_{1} d \beta_{2} d \gamma
$$

whenever $n \rightarrow \infty$, for any $\varphi \in C_{0}^{\infty}\left(D_{4} \times(0, T)\right)$ and $\phi \in\left(C_{0}^{\infty}\left(D_{4} \times(0, T)\right)\right)^{2}$.

Proof. Let $\varphi \in C_{0}^{\infty}\left(D_{4} \times(0, T)\right)$ and

$$
c_{n}\left(t, x, y, \alpha_{1}, \alpha_{2}\right)=\delta\left((x, y)-\left(x_{n}, y_{n}\right)\left(t, \alpha_{1}, \alpha_{2}\right)\right)
$$

then, we have

$$
\begin{aligned}
\left\langle\varphi, c_{n}\right\rangle=\int_{D_{2}} \int_{0}^{T} \varphi\left(x_{n}\left(t, \alpha_{1}, \alpha_{2}\right), y_{n}\left(t, \alpha_{1}, \alpha_{2}\right), \alpha_{1}, \alpha_{2}, t\right) d t d \alpha_{1} d \alpha_{2} \\
=\sum_{i=0}^{n-1} \int_{\frac{2 \pi}{n} i}^{\frac{2 \pi}{n}(i+1)} \int_{0}^{2 \pi} \int_{0}^{T} \varphi\left(x_{n}^{i}\left(t, \alpha_{1}, \alpha_{2}\right), y_{n}^{i}\left(t, \alpha_{1}, \alpha_{2}\right), \alpha_{1}, \alpha_{2}, t\right) d t d \alpha_{1} d \alpha_{2} \\
=\sum_{i=0}^{n-1} \int_{\frac{2 \pi}{n} i}^{\frac{2 \pi}{n}(i+1)} \int_{0}^{2 \pi} \int_{0}^{T} \varphi\left(x\left(t, \alpha_{1}, n\left(\alpha_{2}-\frac{2 \pi i}{n}\right)\right),\right. \\
\left.\quad \frac{1}{n} y\left(t, \alpha_{1}, n\left(\alpha_{2}-\frac{2 \pi i}{n}\right)\right)+\frac{2 \pi i}{n}, \alpha_{1}, \alpha_{2}, t\right) d t d \alpha_{1} d \alpha_{2}
\end{aligned}
$$


Now, make $\beta_{2}=n\left(\alpha_{2}-\frac{2 \pi i}{n}\right)=n \alpha_{2}-2 \pi i$, namely, $\alpha_{2}=\frac{\beta_{2}}{n}+\frac{2 \pi i}{n}$. Then, we have

$$
\begin{aligned}
\left\langle\varphi, c_{n}\right\rangle= & \sum_{i=0}^{n-1} \int_{D_{2}} \int_{0}^{T} \varphi\left(x\left(t, \alpha_{1}, \beta_{2}\right), \frac{1}{n} y\left(t, \alpha_{1}, \beta_{2}\right)+\frac{2 \pi i}{n},\right. \\
& \left.\alpha_{1} \frac{\beta_{2}}{n}+\frac{2 \pi i}{n}, t\right) d t d \alpha_{1} \frac{d \beta_{2}}{n} \\
= & A_{n}+B_{n},
\end{aligned}
$$

where

$$
A_{n}=\frac{1}{2 \pi} \sum_{i=0}^{n-1}\left(\int_{D_{2}} \int_{0}^{T} \varphi\left(x\left(t, \alpha_{1}, \beta_{2}\right), \frac{2 \pi i}{n}, \alpha_{1}, \frac{2 \pi i}{n}, t\right) d t d \alpha_{1} d \beta_{2}\right) \frac{2 \pi}{n}
$$

and

$$
\begin{aligned}
B_{n}= & \sum_{i=0}^{n-1} \frac{1}{n} \int_{D_{2}} \int_{0}^{T}\left[\varphi\left(x\left(t, \alpha_{1}, \beta_{2}\right), \frac{1}{n} y\left(t, \alpha_{1}, \beta_{2}\right)+\frac{2 \pi i}{n}, \alpha_{1}, \frac{\beta_{2}}{n}+\frac{2 \pi i}{n}, t\right)\right. \\
& \left.-\varphi\left(x\left(t, \alpha_{1}, \beta_{2}\right), \frac{2 \pi i}{n}, \alpha_{1}, \frac{2 \pi i}{n}, t\right)\right] d t d \alpha_{1} d \beta_{2} .
\end{aligned}
$$

Note that, by Mean Value Theorem, we have

$$
\begin{gathered}
\mid \varphi\left(x\left(t, \alpha_{1}, \beta_{2}\right), \frac{1}{n} y\left(t, \alpha_{1}, \beta_{2}\right)+\frac{2 \pi i}{n}, \alpha_{1}, \frac{\beta_{2}}{n}+\frac{2 \pi i}{n}, t\right)- \\
-\varphi\left(x\left(t, \alpha_{1}, \beta_{2}\right), \frac{2 \pi i}{n}, \alpha_{1}, \frac{2 \pi i}{n}, t\right)|=| \frac{\partial \varphi}{\partial y} \frac{1}{n} y\left(t, \alpha_{1}, \alpha_{2}\right)+\frac{\partial \varphi}{\partial \beta_{2}} \frac{\beta_{2}}{n} \mid \leq \\
\leq\left(\left\|\frac{\partial \varphi}{\partial y}\right\|_{L^{\infty}\left((0,2 \pi)^{4} \times(0, T)\right)} \frac{\left|y\left(t, \alpha_{1}, \alpha_{2}\right)\right|}{n}+\left\|\frac{\partial \varphi}{\partial \beta_{2}}\right\|_{L^{\infty}\left((0,2 \pi)^{4} \times(0, T)\right)} \frac{\left|\beta_{2}\right|}{n}\right) \leq \\
\leq \frac{2 \pi}{n}\left(\left\|\frac{\partial \varphi}{\partial y}\right\|_{L^{\infty}}+\left\|\frac{\partial \varphi}{\partial \beta_{2}}\right\|_{L^{\infty}}\right) \leq \frac{2 \pi}{n}\|D \varphi\|_{L^{\infty}} .
\end{gathered}
$$

Then, we obtain

$$
\begin{aligned}
\left|B_{n}\right| \leq & \sum_{i=0}^{n-1} \frac{1}{n} \int_{D_{2}} \int_{0}^{T} \mid\left[\varphi\left(x\left(t, \alpha_{1}, \beta_{2}\right), \frac{1}{n} y\left(t, \alpha_{1}, \beta_{2}\right)+\frac{2 \pi i}{n}, \alpha_{1}, \frac{\beta_{2}}{n}+\frac{2 \pi i}{n}, t\right)\right. \\
& \left.-\varphi\left(x\left(t, \alpha_{1}, \beta_{2}\right), \frac{2 \pi i}{n}, \alpha_{1}, \frac{2 \pi i}{n}, t\right)\right] \mid d t d \alpha_{1} d \beta_{2} \\
\leq & \sum_{i=0}^{n-1} \frac{1}{n} \frac{2 \pi}{n}\|D \varphi\|_{L^{\infty}} \int_{0}^{2 \pi} \int_{0}^{2 \pi} \int_{0}^{T} d t d \alpha_{1} d \beta_{2} \\
= & \frac{2 \pi}{n}\|D \varphi\|_{L^{\infty}} 4 \pi^{2} T-\frac{2 \pi}{n^{2}}\|D \varphi\|_{L^{\infty}} 4 \pi^{2} T \\
\leq & \frac{2 \pi}{n}\|D \varphi\|_{L^{\infty}} 4 \pi^{2} T .
\end{aligned}
$$


Therefore, $B_{n} \rightarrow 0$, when $n \rightarrow \infty$.

Now, define the function $\psi$ by

$$
\psi(\gamma):=\int_{D_{2}} \int_{0}^{T} \varphi\left(x\left(t, \alpha_{1}, \beta_{2}\right), \gamma, \alpha_{1}, \gamma, t\right) d t d \alpha_{1} d \beta_{2}
$$

thus, we rewrite (3.5) as

$$
A_{n}=\frac{1}{2 \pi} \sum_{i=0}^{n-1} \psi\left(\frac{2 \pi i}{n}\right) \frac{2 \pi}{n}=\frac{1}{2 \pi} \sum_{i=0}^{n-1} \psi\left(\frac{2 \pi i}{n}\right)\left(\frac{2 \pi(i+1)}{n}-\frac{2 \pi i}{n}\right) .
$$

By this form, if $\gamma_{i}=\frac{2 \pi i}{n}$ then, $\left\{\gamma_{0}, \gamma_{1}, \cdots, \gamma_{n}\right\}$ is a partition of the $(0,2 \pi)$ and $A_{n}=$ $\frac{1}{2 \pi} \sum_{i=0}^{n-1} \psi\left(\gamma_{i}\right)\left(\gamma_{i+1}-\gamma_{i}\right)$ is a Riemann sum. Therefore,

$$
A_{n} \rightarrow \frac{1}{2 \pi} \int_{0}^{2 \pi} \psi(\gamma) d \gamma
$$

when $n \rightarrow \infty$. Then, we conclude that

$$
\left\langle\varphi, c_{n}\right\rangle \rightarrow \frac{1}{2 \pi} \int_{D_{3}} \int_{0}^{T} \varphi\left(x\left(t, \alpha_{1}, \beta_{2}\right), \gamma, \alpha_{1}, \gamma, t\right) d t d \alpha_{1} d \beta_{2} d \gamma,
$$

when $n \rightarrow \infty$, for any $\varphi \in C_{0}^{\infty}\left(D_{4} \times(0, T)\right)$.

Now, consider $\phi \in\left(C_{0}^{\infty}\left(D_{4} \times(0, T)\right)\right)^{2}$ and let

$$
\begin{aligned}
m_{n} & =u_{n}(x, y) \delta\left((x, y)-\left(x_{n}\left(t, \alpha_{1}, \alpha_{2}\right), y_{n}\left(t, \alpha_{1}, \alpha_{2}\right)\right)\right) \\
& =\left(u_{1}^{1}(x, n y), \frac{1}{n} u_{1}^{2}(x, n y)\right) \delta\left((x, y)-\left(x_{n}\left(t, \alpha_{1}, \alpha_{2}\right), y_{n}\left(t, \alpha_{1}, \alpha_{2}\right)\right)\right) .
\end{aligned}
$$

Then, we obtain

$$
\begin{aligned}
\left\langle\phi, m_{n}\right\rangle= & \int_{D_{2}} \int_{0}^{T} \phi\left(x_{n}\left(t, \alpha_{1}, \alpha_{2}\right), y_{n}\left(t, \alpha_{1}, \alpha_{2}\right), \alpha_{1}, \alpha_{2}, t\right)\left[u _ { 1 } ^ { 1 } \left(x_{n}\left(t, \alpha_{1}, \alpha_{2}\right),\right.\right. \\
& \left.\left.n y_{n}\left(t, \alpha_{1}, \alpha_{2}\right)\right), \frac{1}{n} u_{1}^{2}\left(x_{n}\left(t, \alpha_{1}, \alpha_{2}\right), n y_{n}\left(t, \alpha_{1}, \alpha_{2}\right)\right)\right] d t d \alpha_{1} d \alpha_{2} \\
= & \sum_{i=0}^{n-1} \int_{\frac{2 \pi}{n} i}^{\frac{2 \pi}{n}(i+1)} \int_{0}^{2 \pi} \int_{0}^{T} \phi\left(x_{n}^{i}\left(t, \alpha_{1}, \alpha_{2}\right), y_{n}^{i}\left(t, \alpha_{1}, \alpha_{2}\right), \alpha_{1}, \alpha_{2}, t\right) \\
& {\left[u_{1}^{1}\left(x_{n}^{i}\left(t, \alpha_{1}, \alpha_{2}\right), n y_{n}^{i}\left(t, \alpha_{1}, \alpha_{2}\right)\right), \frac{1}{n} u_{1}^{2}\left(x_{n}^{i}\left(t, \alpha_{1}, \alpha_{2}\right),\right.\right.} \\
& \left.\left.n y_{n}^{i}\left(t, \alpha_{1}, \alpha_{2}\right)\right)\right] d t d \alpha_{1} d \alpha_{2},
\end{aligned}
$$


and therefore, making $\beta_{2}=n\left(\alpha_{2}-\frac{2 \pi i}{n}\right)$ we have

$$
\begin{aligned}
\left\langle\phi, m_{n}\right\rangle= & \sum_{i=0}^{n-1} \int_{D_{2}} \int_{0}^{T} \frac{1}{n} \phi^{1}\left(x\left(t, \alpha_{1}, \beta_{2}\right), \frac{1}{n} y\left(t, \alpha_{1}, \alpha_{2}\right)+\frac{2 \pi i}{n}, \alpha_{1}, \frac{\beta_{2}}{n}+\frac{2 \pi i}{n}, t\right) \\
& u_{1}^{1}\left(x\left(t, \alpha_{1}, \beta_{2}\right), y\left(t, \alpha_{1}, \beta_{2}\right)\right) d t d \alpha_{1} d \beta_{2}+ \\
& +\sum_{i=0}^{n-1} \int_{D_{2}} \int_{0}^{T} \frac{1}{n^{2}} \phi^{2}\left(x\left(t, \alpha_{1}, \beta_{2}\right), \frac{1}{n} y\left(t, \alpha_{1}, \alpha_{2}\right)+\frac{2 \pi i}{n}, \alpha_{1}, \frac{2 \pi i}{n}, t\right) \\
& u_{1}^{2}\left(x\left(t, \alpha_{1}, \beta_{2}\right), y\left(t, \alpha_{1}, \beta_{2}\right)\right) d t d \alpha_{1} d \beta_{2} \\
= & A_{n}^{1}+B_{n}^{1}+A_{n}^{2}+B_{n}^{2},
\end{aligned}
$$

where,

$$
\begin{gathered}
A_{n}^{1}=\frac{1}{2 \pi} \sum_{i=0}^{n-1}\left(\int_{D_{2}} \int_{0}^{T} \frac{1}{n} \phi^{1}\left(x\left(t, \alpha_{1}, \beta_{2}\right), \frac{2 \pi i}{n}, \alpha_{1}, \frac{2 \pi i}{n}, t\right)\right. \\
\left.u_{1}^{1}\left(x\left(t, \alpha_{1}, \beta_{2}\right), y\left(t, \alpha_{1}, \beta_{2}\right)\right) d t d \alpha_{1} d \beta_{2}\right) 2 \pi, \\
A_{n}^{2}=\frac{1}{2 \pi n} \sum_{i=0}^{n-1}\left(\int_{D_{2}} \int_{0}^{T} \frac{1}{n} \phi^{2}\left(x\left(t, \alpha_{1}, \beta_{2}\right), \frac{2 \pi i}{n}, \alpha_{1}, \frac{2 \pi i}{n}, t\right)\right. \\
\left.u_{1}^{2}\left(x\left(t, \alpha_{1}, \beta_{2}\right), y\left(t, \alpha_{1}, \beta_{2}\right)\right) d t d \alpha_{1} d \beta_{2}\right) 2 \pi, \\
B_{n}^{1}=\sum_{i=0}^{n-1} \int_{D_{2}} \int_{0}^{T} \frac{1}{n}\left[\phi ^ { 1 } \left(x\left(t, \alpha_{1}, \beta_{2}\right), \frac{1}{n} y\left(t, \alpha_{1}, \beta_{2}\right)+\frac{2 \pi i}{n},\right.\right. \\
\left.\left.B_{n}^{2}, \frac{\beta_{2}}{n}+\frac{2 \pi i}{n}, t\right)-\phi^{1}\left(x\left(t, \alpha_{1}, \beta_{2}\right), \frac{2 \pi i}{n}, \alpha_{1}, \frac{2 \pi i}{n}, t\right)\right] \\
\sum_{i=0}^{1} \int_{D_{2}} \int_{0}^{T}\left(x\left(t, \alpha_{1}, \beta_{2}\right), y\left(t, \alpha_{1}, \beta_{2}\right)\right) d t d \alpha_{1} d \beta_{2}, \\
\quad-\phi^{2}\left(x\left(t, \alpha_{1}, \beta_{2}\right), \frac{2 \pi i}{n}, \phi_{1}\left(x, \frac{2 \pi i}{n}, t\right)\right] u_{1}^{2}\left(x\left(t, \alpha_{1}, \beta_{2}\right), y\left(t, \alpha_{1}, \beta_{2}\right)\right) d t d \alpha_{1} d \beta_{2},
\end{gathered}
$$

As we seen before, we conclude that

$$
\begin{gathered}
\mid \phi^{i}\left(x\left(t, \alpha_{1}, \beta_{2}\right), \frac{1}{n} y\left(t, \alpha_{1}, \beta_{2}\right)+\frac{2 \pi i}{n}, \alpha_{1}, \frac{\beta_{2}}{n}+\frac{2 \pi i}{n}, t\right)- \\
-\phi^{i}\left(x\left(t, \alpha_{1}, \beta_{2}\right), \frac{2 \pi i}{n}, \alpha_{1}, \frac{2 \pi i}{n}, t\right) \mid \leq \frac{2 \pi}{n}\|D \phi\|_{L^{\infty}}, \quad i=1,2 .
\end{gathered}
$$


Thus, we have the estimate

$$
\begin{aligned}
\left|B_{n}^{1}\right| \leq & \sum_{i=0}^{n-1} \frac{1}{n} \int_{D_{2}} \int_{0}^{T} \mid \phi^{1}\left(x\left(t, \alpha_{1}, \beta_{2}\right), \frac{1}{n} y\left(t, \alpha_{1}, \beta_{2}\right)+\frac{2 \pi i}{n}, \alpha_{1}, \frac{\beta_{2}}{n}+\frac{2 \pi i}{n}, t\right) \\
& -\phi^{1}\left(x\left(t, \alpha_{1}, \beta_{2}\right), \frac{2 \pi i}{n}, \alpha_{1}, \frac{2 \pi i}{n}, t\right)|| u_{1}^{1}\left(x\left(t, \alpha_{1}, \beta_{2}\right), y\left(t, \alpha_{1}, \beta_{2}\right)\right) \mid d t d \alpha_{1} d \beta_{2}, \\
\leq & \frac{2 \pi}{n}\|D \phi\|_{L^{\infty}} 4 \pi^{2} T C .
\end{aligned}
$$

Therefore, $B_{n}^{1} \rightarrow 0$, when $n \rightarrow \infty$. Now, we go to study the term $B_{n}^{2}$.

$$
\begin{aligned}
\left|B_{n}^{2}\right| \leq & \sum_{i=0}^{n-1} \frac{1}{n^{2}} \int_{D_{2}} \int_{0}^{T} \mid \phi^{2}\left(x\left(t, \alpha_{1}, \beta_{2}\right), \frac{1}{n} y\left(t, \alpha_{1}, \beta_{2}\right)+\frac{2 \pi i}{n}, \alpha_{1}, \frac{\beta_{2}}{n}+\frac{2 \pi i}{n}, t\right) \\
& -\phi^{2}\left(x\left(t, \alpha_{1}, \beta_{2}\right), \frac{2 \pi i}{n}, \alpha_{1}, \frac{2 \pi i}{n}, t\right)|| u_{1}^{2}\left(x\left(t, \alpha_{1}, \beta_{2}\right), y\left(t, \alpha_{1}, \beta_{2}\right)\right) \mid d t d \alpha_{1} d \beta_{2} \\
\leq & \frac{2 \pi}{n^{2}}\|D \phi\|_{L^{\infty}} 4 \pi^{2} T C,
\end{aligned}
$$

and, therefore, also $B_{n}^{2} \rightarrow 0$, when $n \rightarrow \infty$.

Defining the function $\psi$ by

$$
\psi^{i}(\gamma):=\int_{D_{2}} \int_{0}^{T} \phi^{i}\left(x\left(t, \alpha_{1}, \beta_{2}\right), \gamma, \alpha_{1}, \gamma, t\right) u_{1}^{i}\left(x\left(t, \alpha_{1}, \beta_{2}\right), y\left(t, \alpha_{1}, \beta_{2}\right)\right) d t d \alpha_{1} d \beta_{2},
$$

$i=1,2$, we obtain,

$$
\begin{aligned}
A_{n}^{1} & =\frac{1}{2 \pi} \sum_{i=0}^{n-1} \psi^{1}\left(\frac{2 \pi i}{n}\right) \frac{2 \pi}{n} \\
& =\frac{1}{2 \pi} \sum_{i=0}^{n-1} \psi^{1}\left(\frac{2 \pi i}{n}\right)\left(\frac{2 \pi(i+1)}{n}-\frac{2 \pi i}{n}\right) .
\end{aligned}
$$

By this form, if $\gamma_{i}=\frac{2 \pi i}{n}$ then, $\left\{\gamma_{0}, \gamma_{1}, \cdots, \gamma_{n}\right\}$ is a partition of the $(0,2 \pi)$ and $A_{n}^{1}=$ $\frac{1}{2 \pi} \sum_{i=0}^{n-1} \psi^{1}\left(\gamma_{i}\right)\left(\gamma_{i+1}-\gamma_{i}\right)$ is a Riemann sum. Therefore,

$$
A_{n}^{1} \rightarrow \frac{1}{2 \pi} \int_{0}^{2 \pi} \psi^{1}(\gamma) d \gamma, \text { when } \mathrm{n} \rightarrow \infty .
$$

For the last term, we have that

$$
A_{n}^{2}=\frac{1}{2 \pi n} \sum_{i=0}^{n-1} \psi^{2}\left(\frac{2 \pi i}{n}\right) \frac{2 \pi}{n}
$$




$$
=\frac{1}{2 \pi n} \sum_{i=0}^{n-1} \psi^{2}\left(\frac{2 \pi i}{n}\right)\left(\frac{2 \pi(i+1)}{n}-\frac{2 \pi i}{n}\right),
$$

and therefore, $A_{n}^{2} \rightarrow 0$, when $n \rightarrow \infty$.

Then, we conclude that

$\left\langle\phi, m_{n}\right\rangle \rightarrow$

$$
\frac{1}{2 \pi} \int_{D_{3}} \int_{0}^{T} \phi^{1}\left(x\left(t, \alpha_{1}, \beta_{2}\right), \gamma, \alpha_{1}, \gamma, t\right) u_{1}^{1}\left(x\left(t, \alpha_{1}, \beta_{2}\right), y\left(t, \alpha_{1}, \beta_{2}\right)\right) d t d \alpha_{1} d \beta_{2} d \gamma
$$

when $n \rightarrow \infty$, for any $\phi \in\left(C_{0}^{\infty}\left(D_{4} \times(0, T)\right)\right)^{2}$.

By the last theorem we can conclude that

$$
\left\langle\varphi, c_{n}\right\rangle \rightarrow \frac{1}{2 \pi} \int_{D_{3}} \int_{0}^{T} \varphi\left(x\left(t, \alpha_{1}, \beta_{2}\right), \gamma, \alpha_{1}, \gamma, t\right) d t d \alpha_{1} d \beta_{2} d \gamma
$$

and $\left\langle\phi, m_{n}\right\rangle \rightarrow$

$$
\frac{1}{2 \pi} \int_{D_{3}} \int_{0}^{T} \phi^{1}\left(x\left(t, \alpha_{1}, \beta_{2}\right), \gamma, \alpha_{1}, \gamma, t\right) u_{1}^{1}\left(x\left(t, \alpha_{1}, \beta_{2}\right), y\left(t, \alpha_{1}, \beta_{2}\right)\right) d t d \alpha_{1} d \beta_{2} d \gamma,
$$

whenever $n \rightarrow \infty$, for any

$$
\varphi \in C_{0}^{\infty}\left(D_{4} \times(0, T)\right) \text { and } \phi \in\left(C_{0}^{\infty}\left(D_{4} \times(0, T)\right)\right)^{2} \text {. }
$$

Now, note that

$$
2 \pi\left\langle\varphi, c_{n}\right\rangle \rightarrow \int_{D_{3}} \int_{0}^{T} \varphi\left(x\left(t, \alpha_{1}, \beta_{2}\right), \gamma, \alpha_{1}, \gamma, t\right) d t d \alpha_{1} d \beta_{2} d \gamma
$$

is equivalent to

$$
\int_{0}^{2 \pi}\left\langle\varphi, c_{n}\right\rangle d \beta_{2} \rightarrow \int_{D_{3}} \int_{0}^{T} \varphi\left(x\left(t, \alpha_{1}, \beta_{2}\right), \gamma, \alpha_{1}, \gamma, t\right) d t d \alpha_{1} d \gamma d \beta_{2}
$$

and, therefore,

$$
\int_{0}^{2 \pi}\left[\left\langle\varphi, c_{n}\right\rangle-\int_{D_{2}} \int_{0}^{T} \varphi\left(x\left(t, \alpha_{1}, \beta_{2}\right), \gamma, \alpha_{1}, \gamma, t\right) d t d \alpha_{1} d \gamma\right] d \beta_{2} \rightarrow 0
$$

Then, we conclude that

$$
\left\langle\varphi, c_{n}\right\rangle \rightarrow \int_{D_{2}} \int_{0}^{T} \varphi\left(x\left(t, \alpha_{1}, \beta_{2}\right), \gamma, \alpha_{1}, \gamma, t\right) d t d \alpha_{1} d \gamma
$$


Of completely analogous way, we also conclude that

$\left\langle\varphi, m_{n}\right\rangle \rightarrow$

$$
\int_{D_{2}} \int_{0}^{T} \phi^{1}\left(x\left(t, \alpha_{1}, \beta_{2}\right), \gamma, \alpha_{1}, \gamma, t\right) u_{1}^{1}\left(x\left(t, \alpha_{1}, \beta_{2}\right), y\left(t, \alpha_{1}, \beta_{2}\right)\right) d t d \alpha_{1} d \gamma
$$

Thus, the limite $(c, m)$ is given by

$$
\left\{\begin{array}{l}
c\left(x, y, \alpha_{1}, \alpha_{2}, t\right)=\delta\left(\left(x, \alpha_{2}\right)-\left(x\left(t, \alpha_{1}, \beta_{2}\right), y\right)\right) \\
m\left(x, y, \alpha_{1}, \alpha_{2}, t\right)=\delta\left(\left(x, \alpha_{2}\right)-\left(x\left(t, \alpha_{1}, \beta_{2}\right), y\right)\right)\left(u_{1}^{1}\left(x, y\left(t, \alpha_{1}, \beta_{2}\right)\right), 0\right) .
\end{array}\right.
$$

\section{Solution to the Relaxed Euler Equations}

In this section we will conclude our work showing that the pair $(c, m)$, build in the before section, satisfy the relaxed Euler equations.

Theorem 4.1. The pair of measures $(c, m)$ defined in (3.6) satisfy the following equations

$$
\int_{D_{2}} c\left(t, x, y, \alpha_{1}, \alpha_{2}\right) d \alpha_{1} d \alpha_{2}=1, \quad \partial_{t} c+\nabla \cdot m=0, \quad \partial_{t}(c v)+\nabla \cdot(c v \otimes v)+c \nabla p=0
$$

in the sense of distributions.

Proof. Note that

$$
\langle 1, c\rangle=\int_{D_{2}} \int_{0}^{T} d t d \alpha_{1} d \alpha_{2}=\int_{D_{2}} \int_{0}^{T} d t d x d y
$$

Then, we obtain

$$
\int_{D_{2}} \int_{0}^{T}\left(\int_{D_{2}} c\left(t, x, y, \alpha_{1}, \alpha_{2}\right) d \alpha_{1} d \alpha_{2}-1\right) d t d x d y=0
$$

and, therefore, $\int_{D_{2}} c\left(t, x, y, \alpha_{1}, \alpha_{2}\right) d \alpha_{1} d \alpha_{2}=1$.

Now, we will show that pair $(c, m)$ satisfy the equation $\partial_{t} c+\nabla \cdot m=0$. Consider $\varphi \in$ $C_{0}^{\infty}\left(D_{4} \times(0, T)\right)$, thus

$$
\begin{aligned}
& \left\langle\varphi\left(x, y, \alpha_{1}, \alpha_{2}, t\right), \partial_{t} c\left(t, x, y, \alpha_{1}, \alpha_{2}\right)+\nabla_{(x, y)} \cdot m\left(t, x, y, \alpha_{1}, \alpha_{2}\right)\right\rangle= \\
= & -\int_{D_{2}} \int_{0}^{T} \partial_{t} \varphi\left(x\left(t, \alpha_{1}, \beta_{2}\right), y, \alpha_{1}, y, t\right) d t d \alpha_{1} d y \\
& -\int_{D_{2}} \int_{0}^{T} \partial_{x} \varphi\left(x\left(t, \alpha_{1}, \beta_{2}\right), y, \alpha_{1}, y, t\right) u_{1}^{1}\left(x\left(t, \alpha_{1}, \beta_{2}\right), y\left(t, \alpha_{1}, \beta_{2}\right)\right) d t d \alpha_{1} d y
\end{aligned}
$$




$$
\begin{aligned}
& =-\int_{D_{2}} \int_{0}^{T} \partial_{t}\left(\varphi\left(x\left(t, \alpha_{1}, \beta_{2}\right), y, \alpha_{1}, y, t\right)\right) d t d \alpha_{1} d y \\
& =-\int_{D_{2}}\left(\varphi\left(\alpha_{1}, y, \alpha_{1}, y, T\right)-\varphi\left(\beta_{2}, y, \alpha_{1}, y, 0\right)\right) d \alpha_{1} d y=0 .
\end{aligned}
$$

Finally, we will show that the pair $(c, m)$ also satisfy the equation $\partial_{t}(c v)+\nabla \cdot(c v \otimes v)+c \nabla p=$ 0 . First note that $\left[d i v_{(x, y)}(u \otimes u)\right]^{i}=u^{i} \operatorname{div}_{(x, y)} u+u \cdot \nabla_{(x, y)} u^{i}=u \cdot \nabla_{(x, y)} u^{i}$, because $d i v_{(x, y)} u=0$. Let $\varphi \in\left(C_{0}^{\infty}\left(D_{4} \times(0, T)\right)\right)^{2}$, then

$$
\begin{aligned}
\left\langle\varphi^{1}\left(x, y, \alpha_{1}, \alpha_{2}, t\right), \partial_{t}\left(c\left(t, x, y, \alpha_{1}, \alpha_{2}\right) v^{1}\left(x, y, \alpha_{1}, \alpha_{2}, t\right)\right\rangle+\right. & \\
+ & \left\langle\varphi^{1}\left(x, y, \alpha_{1}, \alpha_{2}, t\right), \nabla_{(x, y)} \cdot\left(c\left(t, x, y, \alpha_{1}, \alpha_{2}\right) v\left(x, y, \alpha_{1}, \alpha_{2}, t\right) \otimes v\left(x, y, \alpha_{1}, \alpha_{2}, t\right)\right)^{1}\right\rangle \\
& +\left\langle\varphi^{1}\left(x, y, \alpha_{1}, \alpha_{2}, t\right), c\left(x, y, \alpha_{1}, \alpha_{2}, t\right) \partial_{x} p(x, y)\right\rangle= \\
= & -\int_{D_{2}} \int_{0}^{T} u_{1}^{1}\left(x\left(t, \alpha_{1}, \beta_{2}\right), y\left(t, \alpha_{1}, \beta_{2}\right)\right) \partial_{t} \varphi^{1}\left(x\left(t, \alpha_{1}, \beta_{2}\right), y, \alpha_{1}, y, t\right) d t d \alpha_{1} d y \\
& -\int_{D_{2}} \int_{0}^{T}\left[u_{1}^{1}\left(x\left(t, \alpha_{1}, \beta_{2}\right), y\left(t, \alpha_{1}, \beta_{2}\right)\right)\right]^{2} \partial_{x} \varphi^{1}\left(x\left(t, \alpha_{1}, \beta_{2}\right), y, \alpha_{1}, y, t\right) d t d \alpha_{1} d y \\
& +\int_{D_{2}} \int_{0}^{T} \partial_{x} p\left(x\left(t, \alpha_{1}, \beta_{2}\right), y\right) \varphi^{1}\left(x\left(t, \alpha_{1}, \beta_{2}\right), y, \alpha_{1}, y, t\right) d t d \alpha_{1} d y \\
= & -\int_{D_{2}} \int_{0}^{T} u_{1}^{1}\left(x\left(t, \alpha_{1}, \beta_{2}\right), y\left(t, \alpha_{1}, \beta_{2}\right)\right) \partial_{t}\left[\varphi^{1}\left(x\left(t, \alpha_{1}, \beta_{2}\right), y, \alpha_{1}, y, t\right)\right] d t d \alpha_{1} d y \\
& +\int_{D_{2}} \int_{0}^{T}\left[u_{1}^{1}\left(x\left(t, \alpha_{1}, \beta_{2}\right), y\left(t, \alpha_{1}, \beta_{2}\right)\right)\right]^{2} \partial_{x} \varphi^{1}\left(x\left(t, \alpha_{1}, \beta_{2}\right), y, \alpha_{1}, y, t\right) d t d \alpha_{1} d y \\
& -\int_{D_{2}} \int_{0}^{T}\left[u_{1}^{1}\left(x\left(t, \alpha_{1}, \beta_{2}\right), y\left(t, \alpha_{1}, \beta_{2}\right)\right)\right]^{2} \partial_{x} \varphi^{1}\left(x\left(t, \alpha_{1}, \beta_{2}\right), y, \alpha_{1}, y, t\right) d t d \alpha_{1} d y \\
& +\int_{D_{2}} \int_{0}^{T} \partial_{x} p\left(x\left(t, \alpha_{1}, \beta_{2}\right), y\right) \varphi^{1}\left(x\left(t, \alpha_{1}, \beta_{2}\right), y, \alpha_{1}, y, t\right) d t d \alpha_{1} d y \\
= & \int_{D_{2}} \int_{0}^{T} \partial_{t}\left[u_{1}^{1}\left(x\left(t, \alpha_{1}, \beta_{2}\right), y\left(t, \alpha_{1}, \beta_{2}\right)\right)\right] \varphi^{1}\left(x\left(t, \alpha_{1}, \beta_{2}\right), y, \alpha_{1}, y, t\right) d t d \alpha_{1} d y \\
& +\int_{D_{2}} \int_{0}^{T} \partial_{x} p\left(x\left(t, \alpha_{1}, \beta_{2}\right), y\right) \varphi^{1}\left(x\left(t, \alpha_{1}, \beta_{2}\right), y, \alpha_{1}, y, t\right) d t d \alpha_{1} d y \\
= & \int_{D_{2}} \int_{0}^{T}\left[\partial_{x} u_{1}^{1}\left(x\left(t, \alpha_{1}, \beta_{2}\right), y\left(t, \alpha_{1}, \beta_{2}\right)\right) \partial_{t} x\left(t, \alpha_{1}, \beta_{2}\right)\right. \\
& \left.+\partial_{y} u_{1}^{1}\left(x\left(t, \alpha_{1}, \beta_{2}\right), y\left(t, \alpha_{1}, \beta_{2}\right)\right) \partial_{t} y\left(t, \alpha_{1}, \beta_{2}\right)\right] \varphi^{1}\left(x\left(t, \alpha_{1}, \beta_{2}\right), y, \alpha_{1}, y, t\right) d t d \alpha_{1} d y \\
& +\int_{D_{2}} \int_{0}^{T} \partial_{x} p\left(x\left(t, \alpha_{1}, \beta_{2}\right), y\right) \varphi^{1}\left(x\left(t, \alpha_{1}, \beta_{2}\right), y, \alpha_{1}, y, t\right) d t d \alpha_{1} d y \\
& \int_{(x, y)} u_{1}^{1}\left(x\left(t, \alpha_{1}, \beta_{2}\right), y\left(t, \alpha_{1}, \beta_{2}\right)\right)
\end{aligned}
$$




$$
\begin{array}{r}
u_{1}^{1}\left(x\left(t, \alpha_{1}, \beta_{2}\right), y\left(t, \alpha_{1}, \beta_{2}\right)\right) \varphi^{1}\left(x\left(t, \alpha_{1}, \beta_{2}\right), y, \alpha_{1}, y, t\right) d t d \alpha_{1} d y \\
+\int_{D_{2}} \int_{0}^{T} \partial_{x} p\left(x\left(t, \alpha_{1}, \beta_{2}\right), y\right) \varphi^{1}\left(x\left(t, \alpha_{1}, \beta_{2}\right), y, \alpha_{1}, y, t\right) d t d \alpha_{1} d y
\end{array}
$$

Since that $p(x)=-\frac{1}{4} \cos (2 x)$ we have that $\partial_{x} p=\frac{1}{2} \sin (2 x)$ and then,

$$
\partial_{x} p\left(x\left(t, \alpha_{1}, \beta_{2}\right), y\right)=\partial_{x} p\left(x\left(t, \alpha_{1}, \beta_{2}\right), y\left(x\left(t, \alpha_{1}, \beta_{2}\right)\right) .\right.
$$

Therefore, we conclude that

$$
\begin{gathered}
\left\langle\varphi^{1}\left(x, y, \alpha_{1}, \alpha_{2}, t\right), \partial_{t}\left(c\left(t, x, y, \alpha_{1}, \alpha_{2}\right) v^{1}\left(x, y, \alpha_{1}, \alpha_{2}, t\right)\right\rangle+\right. \\
+\left\langle\varphi^{1}\left(x, y, \alpha_{1}, \alpha_{2}, t\right), \nabla_{(x, y)} \cdot\left(c\left(t, x, y, \alpha_{1}, \alpha_{2}\right) v\left(x, y, \alpha_{1}, \alpha_{2}, t\right) \otimes v\left(x, y, \alpha_{1}, \alpha_{2}, t\right)\right)^{1}\right\rangle+ \\
+\left\langle\varphi^{1}\left(x, y, \alpha_{1}, \alpha_{2}, t\right), c\left(x, y, \alpha_{1}, \alpha_{2}, t\right) \partial_{x} p(x, y)\right\rangle= \\
=\int_{D_{2}} \int_{0}^{T}\left[\nabla_{(x, y)} u_{1}^{1}\left(x\left(t, \alpha_{1}, \beta_{2}\right), y\left(t, \alpha_{1}, \beta_{2}\right)\right) u_{1}^{1}\left(x\left(t, \alpha_{1}, \beta_{2}\right), y\left(t, \alpha_{1}, \beta_{2}\right)\right)\right. \\
\left.+\partial_{x} p\left(x\left(t, \alpha_{1}, \beta_{2}\right), y\left(t, \alpha_{1}, \beta_{2}\right)\right)\right] \varphi^{1}\left(x\left(t, \alpha_{1}, \beta_{2}\right), y, \alpha_{1}, y, t\right) d t d \alpha_{1} d y .
\end{gathered}
$$

Now, note that $u_{1}^{1}(x, n y)=-\cos (x) \sin (n y)$ and $u_{1}^{2}(x, n y)=\sin (x) \cos (n y)$, namely, $u_{1}^{1}(x, z)=$ $-\cos (x) \sin (z)$ and $u_{1}^{2}(x, z)=\sin (x) \cos (z)$, where $z=n y$. Then,

$$
\nabla u_{1}^{1} \cdot u_{1}=-\sin (x) \cos (x)=-\frac{1}{2} \sin (2 x),
$$

and, therefore,

$$
\nabla u_{1}^{1} \cdot u_{1}+\partial_{x} p=-\frac{1}{2} \sin (2 x)+\frac{1}{2} \sin (2 x)=0
$$

Then,

$$
\begin{gathered}
\nabla_{(x, y)} u_{1}^{1}\left(x\left(t, \alpha_{1}, \beta_{2}\right), y\left(t, \alpha_{1}, \beta_{2}\right)\right) \cdot u_{1}\left(x\left(t, \alpha_{1}, \beta_{2}\right), y\left(t, \alpha_{1}, \beta_{2}\right)\right)+ \\
+\partial_{x} p\left(x\left(t, \alpha_{1}, \beta_{2}\right), y\left(t, \alpha_{1}, \beta_{2}\right)\right)=0
\end{gathered}
$$

and we can conclude that the pair of measures $(c, m=c v)$ satisfy

$$
\partial_{t}(c v)+\nabla \cdot(c v \otimes v)+c \nabla p=0 .
$$

\section{Acknowledgement.}

The author would like to thank Lucas C. F. Ferreira, Helena J. Nussenzveig Lopes and Milton C. Lopes Filho for their useful comments and Weber F. Pereira for many fruitful discussions. The author gratefully acknowledge FAPESP Thematic Project \#2007/51490-7. 


\section{References}

[1] Arnold, V.I., Sur la Géométrie Différentielle des Groupes de Lie de Dimension Infine et ses Applications à L'Hidrodynamique, Ann. Inst. Fourier, 16, 319-361, 1966.

[2] Arnold, V.I. And Khesin, B., Topological Methods in Hidrodynamics, Annu. Rev. Fluid Mech., 24, 145-166, 1992.

[3] Bensow, R.E., Larson, M.G. And Vesterlund, P., Vorticity-strain residual-based turbulence modelling of the Taylor-Green vortex, Int. J. Numer. Meth. Fluids, 54, 745$756,2007$.

[4] Brenier, Y., The Least Action Principle and the Related Concept of Generalized Flows for Incompressible Perfect Fluids, Journal of the American Mathematical Society, Vol. 2, Number 2, 225-255, 1989.

[5] Brenier, Y., The Dual Least Action Problem for an Ideal, Incompressible Fluid, Arch. Rational Mech. Anal., 122, Number 4, 323-351, 1993.

[6] Brenier, Y., Minimal Geodesics on Groups of Volume-Preserving Maps and Generalized Solutions of the Euler Equations, CPAM 52, 411-452, 1999.

[7] DiPerna, R. AND MAJdA A., Oscillations and Concentrations in Weak Solutions of the Incompressible Fluid Equations, Comm. Math. Phys., 108, 667-689, 1987.

[8] Don, W.-S, Gottlieb, D., Jameson, L., Schilling, O. AND Shu, C.-W., Numerical Convergence Study of Nearly-Incompressible, Inviscid Taylor-Green Vortex Flow, Journal of Scientific Computing, 24, 569-595, 2005.

[9] Ebin, D.G. AND MARsden J., Groups of Diffeomorphisms and the Motion of an Incompressible Fluid, Ann. of Math., 92, 102-163, 1970.

[10] LeE,H. AND Sohrab S.H., Hydrodynamic Aspects of Premixed Flame Stripes in TwoDimensional Stagnation-Point Flows, Combustionand Flame, 101, 441-451, 1995.

[11] Precioso, J.C., Equações relaxadas para hidrodinâmica ideal, não homogênea, Tese de doutorado, IMECC-UNICAMP, 2005.

[12] Shnirelman, A.I., The Geometry of the Groups of Diffeomorphisms and the Dynamics of an Ideal Incompressible Fluid, Mat. Sb (N.S.) 128(170), 82-109, 144, 1985.

[13] Shnirelman, A.I., Generalized Fluid Flows, their Aproximation and Applications, Geom. Funct. Anal., 4, 586-620, 1994.

[14] Shu, C.-W. And Weinan, E., A Numerical Resolution Study of High Order Essentially Non-Oscillatory Schemes Applied to Incompressible flow, Journal of Computational Physics, 110, 39-46, 1994. 
[15] Tartar, L., The Compensated Compactness Method Applied to Systems of Conservation Laws. Systems of Nonlinear Partial Differential Equations, (Oxford, 1982), 263-285. NATO Adv. Sci. Inst. Ser. C: Math. Phys. Sci., 111. Reidel, Dordrecht-Boston, 1983.

[16] WAN, D.C., WeI, G.W. AND ZHOU, Y.C., Numerical solution of incompressible flows by discrete singular convolution, Int. J. Numer. Meth. Fluids, 38, 789-810, 2002.

[17] WEI, D.C., WEI, A new algorithm for solving some mechanical problems, Comput. Methods Appl. Mech. Engrg., 190, 2017-2030, 2001.

[18] Young, L.C., Lectures on the Calculus of Variations and Optimal Control Theory, Chelsea, New York, 1980. 